Скопје, Македонија

\title{
COMPARISON OF DIFFERENT NUMERICAL METHODS FOR FRACTIONAL DIFFERENTIAL EQUATIONS
}

\author{
YLLDRITA SEFERI, GJORGJI MARKOSKI, AND ALEKSANDAR GJURCHINOVSKI
}

\begin{abstract}
The dynamical properties of fractional-order systems have attraced increasing attention in recent years. In this paper, numerical solutions of Chen system with fractional-order are given by using three different computational methods: Adams-Bashforth (FAB), Adams-BashforthMoulton (FABM) and Fractional Multistep Differential Transformation method (FMDTM). The fractional derivatives are described in the Caputo sense. Fractional FABM method acts like a predictor-corrector pair which represents an amalgamation between FAB and fractional AdamsMoulton (FAM) methods, and it is compared with FMDTM, which is a semi-numerical method that exploits the power series representation of the solution. The system is shown to display interesting dynamical behavior depending on the system parameters, such as a chaotic behavior, as well as stabilization towards regular periodic motion or equilibrium points. Numerically obtained results are analysed to compare various integration algorithms.
\end{abstract}

\section{INTRODUCTION}

A nonlinear dynamical system is any deterministic system goverened by nonlinear equations, whose general solutions cannot be written as a linear combination of the component particular solutions. These systems are widely investigated in engineering, physcs and mathematics because the real physical systems are inherently nonlinear in nature. What is interesting is that some of those nonlinear systems can be described using fractional differential equations $[5,7,10]$. The advantage of modeling the physical systems by involving

2000 Mathematics Subject Classification. 34C28, 34A08, 74H15.

Key words and phrases. Fractional Chen system, Fractional multistep differential transform method, Fractional Adams-Bashforth method, Fractional Adams-Bashforth-Moulton method. 
fractional derivatives is that fractional calculus is very suitable for accurate description of the properties of various real materials, for better modeling of their mechanical and electrical properties.

Exact solutions of the most of the fractional nonlinear differential equations cannot be expressed in a closed form, thus analytical and numerical methods are used almost without exceptions. In this paper we use three diferent numerical algorithms to find numerical approximation of the solutions of fractional differential equations $[1,2,3,6]$ : fractional Adams-Bashforth method (FAB), fractional Adams-Bashforth-Moulton method (FABM) and fractional Multistep Differential Transform method (FMDTM). The FMDTM method was initilally applied to find series solutions of electric circuit problems, employing a Taylor series expansion. On the other hand, FABM is a numerical method known as a predictor-corrector method, which is based on approximation of the integral operator [7].

In this paper as a model we use the well-known fractional-order Chen system [11]:

$$
\begin{aligned}
& D_{t}^{v} x(t)=a(y(t)-x(t)) \\
& D_{t}^{v} y(t)=(c-a) x(t)-x(t) z(t)+c y(t) \\
& D_{t}^{v} z(t)=x(t) y(t)-b z(t)
\end{aligned}
$$

with $x(0)=c_{1}, y(0)=c_{2}, z(0)=c_{3}$, where $D_{t}^{v}$ is Caputo fractional derivative, $a, b, c$ are real parameters, and $v \in(0,1]$ is the fractional order [12]. For different values of the parameters $a, b$ and $c$, the Chen system (1)-(3) can be regular or chaotic. We would not consider the classical case $(a, b, c)=$ $(35,3,28)$ when the chaotic attractor is shown to exist, but instead choose the parameter values $(a, b, c)=(0.35,3,0.5)$ and see how the numerical integration methods agree with each other for different values of the fractional order $v=$ $0.39, v=0.69$, and $v=0.99$.

To compare the numerically obtained time-series for the three above mentioned computational methods, we use Wolfram Mathematica 11.0 package $[2,3,4,6]$.

\section{Preliminary Definitions}

We first provide some definitions used in the following analysis. The Euler's Gamma functionis defined as:

$$
\Gamma(n)=\int_{0}^{\infty} t^{n-1} e^{-t} d t
$$


Fractional integral of order $v$ for function $f(t)$ can be expressed as follows:

$$
I_{t}^{v} f(t)=D_{t}^{-v}=\frac{1}{\Gamma(v)} \int_{0}^{t}(t-\tau)^{v-1} f(\tau) d \tau
$$

Riemann-Liouville definition of fractional derivative of order $v$ is:

$$
D_{t}^{v} f(t)=\frac{1}{\Gamma(n-v)} \frac{d^{n}}{d t^{n}} \int_{0}^{t}(t-\tau)^{n-v-1} f(\tau) d \tau
$$

Caputo definition of fractional derivative of order $v$ is:

$$
\bar{D}_{t}^{v} f(t)=\frac{1}{\Gamma(n-v)} \int_{0}^{t}(t-\tau)^{n-v-1} f^{(n)}(\tau) d \tau
$$

The choice of using Caputo fractional derivative is based strictly on physical grounds, because it allows initial and boundary conditions to be included in the formulation of the problem. Note that for homogeneous initial conditions, the Riemann-Louville and Caputto operators coincide [10].

\section{NumericAl METHODS}

3.1. Fractional Adams-Bashforth method (FAB). We take the initial value problem

$$
\bar{D}_{t}^{v} y(t)=f(t, y(t)), y^{(k)}(0)=y_{0}^{(k)}
$$

$(k=0,1,2, \ldots,\lceil v\rceil-1)$. In the following we shall always assume that a solution of (8) is sought on some time interval $[0, T]$, and that we have a uniform grid $t_{j}=h j,(j=0,1,2, \ldots, N)$ and a constant time step denoted by $h=\frac{T}{N}$. The goal is to use an approximation scheme to construct approximate solution values $y_{j} \approx y\left(t_{j}\right)$ at the grid points. The most common idea in this context is to apply the (5) operator to the initial value problem (8) thus creating the nonlinear and weakly singular Volterra integral equation of the second kind $[7]$ :

$$
y(t)=\sum_{k=0}^{\lceil v\rceil-1} \frac{y_{0}^{(k)}}{k !} t^{k}+\frac{1}{\Gamma(v)} \int_{0}^{t}(t-\tau)^{v-1} f(\tau, y(\tau)) d \tau
$$

It is well known that the initial value problem (8) is formally equivalent to the Volterra integral equation (9) in the sense that a continuous function is a solution of a given initial value problem if and only if it is a solution of Volterra integral equation. Based on the approximation of the integral operator by the 
product rectangle method, we arrive to the formula $[5,7]$ :

$$
y_{j}=\sum_{k=0}^{\lceil v\rceil-1} \frac{y_{0}^{(k)}}{k !} t_{j}^{k}+h^{v} \sum_{k=0}^{j-1} b_{k, j} f\left(t_{k}, y_{k}\right)
$$

for $(j=0,1,2, \ldots, N)$, where $b_{k, j}$ are the weights defined as:

$$
b_{k, j}=\frac{(j-k)^{v}-(j-k-1)^{v}}{\Gamma(v+1)}
$$

In the limiting case $v \rightarrow 1$ this method reduces to the classical first-order Adams-Bashforth formula that happens to coincide with the forward Euler method.

In the case of the fractional-order Chen system (1)-(3), the algorithm of this method will obtain the following form $[6,3]$ :

$$
\begin{aligned}
& x_{j}=x_{0}+h^{v} \sum_{k=0}^{j-1} b_{k, j} a\left(x_{k}-y_{k}\right) \\
& y_{j}=y_{0}+h^{v} \sum_{k=0}^{j-1} b_{k, j}\left((c-a) x_{k}-x_{k} z_{k}-c y_{k}\right) \\
& z_{j}=z_{0}+h^{v} \sum_{k=0}^{j-1} b_{k, j}\left(x_{k} y_{k}-b z_{k}\right)
\end{aligned}
$$

in the case when $v \in(0,1]$.

3.2. Fractional Adams-Bashforth-Moulton method (FABM). The FAB method is a natural candidate for a predictor in the process of constructing the predictor-corrector FABM method. The Adams-Moulton method can be constructed in similar way like FAB (12)-(14), for the same given initial value problem using the Volterra integral (9) [5]. Based on the approximation of the integral operator (5) by the product triangle method, one obtains the formula:

$$
y_{j}=\sum_{k=0}^{\lceil v\rceil-1} \frac{y_{0}^{(k)}}{k !} t_{j}^{k}+h^{v} \sum_{k=0}^{j-1} a_{k, j} f\left(t_{k}, y_{k}\right)
$$

for $(j=0,1,2, \ldots, N)$, where $a_{k, j}$ are the weights defined as:

$$
a_{k, j}=\left\{\begin{array}{l}
\frac{(j-1)^{v+1}-(j-v-1) j^{v}}{\Gamma(v+2)}, \text { if } j=0 \\
\frac{(j-k+1)^{v+1}+(j-k-1)^{v+1}-2(j-k)^{v+1}}{\Gamma(v+2)}, \\
1, \quad \text { if } j=k
\end{array}\right.
$$

In the limiting case $v \rightarrow 1$ this method reduces to the classical second-order Adams-Moulton formula [3, 6, 9]. 
The above constructed numerical method acts like a corrector in the FABM approximation scheme. The weights $a_{k, j}(16)$ and $b_{k, j}(11)$ essentially have a convolution structure - they only depend on the difference $(j-k)$ of the two indices. This structure allows us to use one-dimensional arrays instead of twodimensional ones, leading to a signficant reduction in the memory requirements during the computation [9].

Taking into account the previous results, the weights (11), (16) respectively, will take the form:

$$
\begin{aligned}
& b[k]=k^{v}-(k-1)^{v} \\
& a[k]=(k+1)^{1+v}+(k-1)^{v+1}-2 k^{v+1}
\end{aligned}
$$

with the main part of the method:

$$
\begin{aligned}
& p=\sum_{k=0}^{\lceil v\rceil-1} \frac{(j h)^{k}}{k !} y_{0}^{(k)}+\frac{h^{v}}{\Gamma(v+1)} \sum_{k=0}^{j-1} b[j-k] f(k h, y[k]) \\
& y[j]=\sum_{k=0}^{\lceil v\rceil-1} \frac{(j h)^{k}}{k !} y_{0}^{(k)}+\frac{h^{v}}{\Gamma(v+2)}\left(f(j h, p)+\left((j-1)^{v+1}-(j-v-1) j^{v}\right) f(0, y[0])+\right. \\
& \left.+\sum_{k=1}^{j-1} a[j-k] f(k h, y[k])\right)
\end{aligned}
$$

In the case of fractional-order Chen system(1)-(3), the method will take the form:

Predictor of the algorithm:

$$
\begin{aligned}
& p[j]=x_{0}+\frac{h^{v}}{\Gamma(v+1)} \sum_{k=0}^{j-1} b[j-k](a(y[k]-x[k])) \\
& q[j]=y_{0}+\frac{h^{v}}{\Gamma(v+1)} \sum_{k=0}^{j-1} b[j-k]((c-a) x[k]-x[k] z[k]+c y[k]) \\
& \left.r[j]=z_{0}+\frac{h^{v}}{\Gamma(v+1)} \sum_{k=0}^{j-1} b[j-k](x[k] y[k]-b z[k])\right)
\end{aligned}
$$

Corrector of the algorithm:

$x[j]=x_{0}+\frac{h^{v}}{\Gamma(v+2)}\left(a(q[j]-p[j])+(j-1)^{v+1}-(j-v-1) j^{v}\right)(a(y[0]-x[0])+$ 


$$
\begin{aligned}
& +\sum_{k=1}^{j-1} a[j-k](a(y[k]-x[k])) \\
& y[j]=y_{0}+\frac{h^{v}}{\Gamma(v+2)}(((c-a) p[j]-p[j] r[j]+c q[j])+ \\
& +\left((j-1)^{v+1}-(j-v-1) j^{v}\right)((c-a) x[0]-x[0] z[0]+c y[0])+ \\
& \left.+\sum_{k=1}^{j-1} a[j-k]((c-a) x[k]-x[k] z[k]+c y[k])\right) \\
& z[j]=z_{0}+\frac{h^{v}}{\Gamma(v+2)}((p[j] q[j]-b r[j])+ \\
& +\left((j-1)^{v+1}-(j-v-1) j^{v}\right)(x[0] y[0]-b z[0])+ \\
& \left.+\sum_{k=1}^{j-1} a[j-k](x[k] y[k]-b z[k])\right)
\end{aligned}
$$

\subsection{Fractional Multistep Differential Transform method (FMDTM).}

The fractional Multistep Differential transform method (FMDTM) is a numerical method based on the Taylor series expansion which constructs an analytical solution in the form of a polynomial. The traditional higher order Taylor series method requires symbolic computation. However, the differential transform method obtains a polynomial series solution by means of an iterative procedure $[1,8]$. First, we seek for the analytical function $f(t)$ in terms of fractional power series as follows:

$$
f(t)=\sum_{k=0}^{\infty} F(k)\left(t-t_{0}\right)^{k v},
$$

where $0<v \leq 1$ is the order of fractional derivative, and $F(k)$ is the fractional differential transform of $f(t)(27)$ given as

$$
F(k)=\frac{1}{\Gamma(v k+1)}\left[\left(\bar{D}_{t_{0}}^{v}\right)^{k}\left(f\left(t_{0}\right)\right)\right] .
$$

Here $\left(\bar{D}_{t_{0}}^{v}\right)^{k}=\bar{D}_{t_{0}}^{v} \cdot \bar{D}_{t_{0}}^{v} \ldots \cdot \bar{D}_{t_{0}}^{v}$ is the $k$-times-differential Caputo fractional derivative $(7)$.

In our application, we will approximate the function $f(t)$ by a truncated series, so the finite form of (27) is:

$$
f(t)=\sum_{k=0}^{N} F(k)\left(t-t_{0}\right)^{k v} .
$$


The following are the basic properties of the Caputo time-fractional derivative and the differential transformation $[1,2,4,8]$ :

(1) Let $f \in C_{-1}^{n}$ then $\bar{D}^{v} f$ for $0 \leq v \leq n$ is well defined and $\bar{D}^{v t_{0}} f \in C_{-1}$

(2) If $f(t)=g(t) \pm h(t)$, then $F(k)=G(k) \pm H(k)$

(3) If $f(t)=g(t) h(t)$, then $F(z)=\sum_{l=0}^{k} G(l) H(k-l)$

(4) If $\left(t-t_{0}\right)^{p}$, then $F(z)=\delta(k-v p)$, where $\delta(k)= \begin{cases}1, & k=0 \\ 0, & k \neq 0\end{cases}$

(5) If $f(t)=\bar{D}^{v t_{0}}[g(t)]$, then $F(k)=\frac{\Gamma(v(k+1)+1)}{\Gamma(v k+1)} G(v+1)$.

We apply the FMDTM for the fractional Chen system, leading to the differential transformation for this system in the form:

$$
\begin{aligned}
& \frac{\Gamma(v(k+1)+1)}{\Gamma(v k+1)} X(k+1)=a(Y[k]-X[k]) \\
& \frac{\Gamma(v(k+1)+1)}{\Gamma(v k+1)} Y(k+1)=(c-a) X(k)-\sum_{l=0}^{k} X(l) Z(k-l)+c Y(k) \\
& \frac{\Gamma(v(k+1)+1)}{\Gamma(v k+1)} Z(k+1)=\sum_{l=0}^{k} X(l) Y(k-l)-b Z(k)
\end{aligned}
$$

where $X(0)=c_{1}, Y(0)=c_{2}, Z(0)=c_{3}$.

The $N$-th order solutions with the inverse transformation are:

$$
\begin{aligned}
& x(t)=\sum_{m=0}^{N} X(m)\left(t-t_{0}\right)^{m v} \\
& y(t)=\sum_{m=0}^{N} Y(m)\left(t-t_{0}\right)^{m v} \\
& z(t)=\sum_{m=0}^{N} Z(m)\left(t-t_{0}\right)^{m v}
\end{aligned}
$$

Numerical results for system (1)-(3) are done using the 10-order solutions with the inverse transformation.

\section{NUMERICAL RESUlts}

The parameters of the fractional-order Chen system are taken $a=0.35$, $b=3$, and $c=0.7$. We aim to show how FAB, FABM and FMDTM methods will agree with each other in the numerical approximation of the solutions of the system. We take the same initial conditions $x(0)=-7, y(0)=0$ and $z(0)=13$ in all the simulations. We consider three different values of the fractional-orders within the interval $(0,1]$, that is $v=0.39, v=0.69$, and 
$v=0.99$. For the FMDTM method we use 10-th order polynomial truncation of the power series. The goal is to compare the resulting time series for each fractional-order, and to investigate the conditions under which they are in good agreement with each other.

In Figs. $1-3$ we plot the time series $x(t), y(t)$ and $z(t)$ of the fractional order Chen system (1)-(3) by using FMDTM (dashed line), FAB (solid line), and FABM (dotted line), for three different values of the order of the fractional derivative: $v=0.39$ (Fig. 1), $v=0.69$ (Fig. 2), and $v=0.99$ (Fig. 3). In all the simulations we immediately observe that the time-series curves for FAB and FABM methods are practically indistinguishable. On the other hand, the time series obtained by FMDTM method are considerably different than the ones obtained by FAB and FABM methods, and this difference is more pronounced as the order of the fractional derivative becomes much less than 1. Particularly, for $v=0.99$ in Fig. 3, we see that the integration curves by FMDTM are very close to the ones obtained by FAB and FABM.

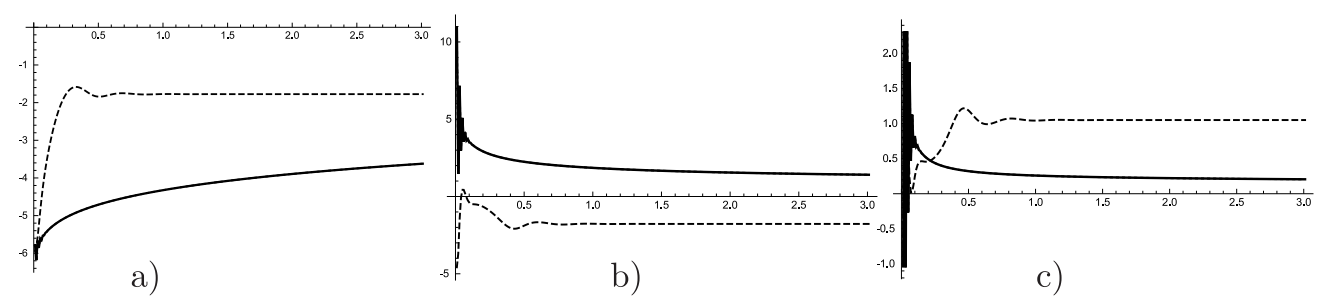

Figure 1. Time-series of the fractional-order Chen system (1)-(3), for $v=0.39$. The step size is $h=0.01$ and $t \in[0,3]$ (FMDTM - dashed line, FAB - solid line, FABM -dotted line]. The integration curves for FAB and FABM are seen as indistinguishable in the given axes scale of the figures. a) time series $x(t)$; b) time series $y(t) ; \mathrm{c})$ time series $z(t)$ 


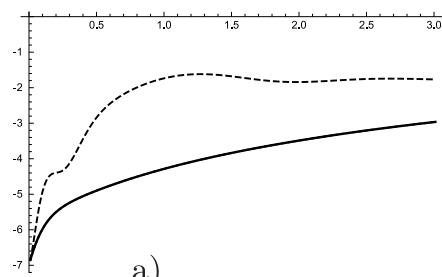

a)

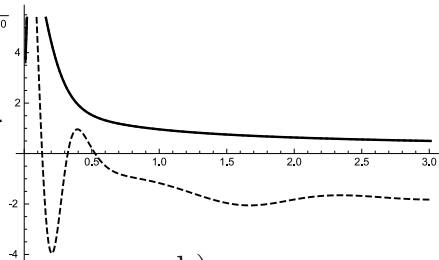

b)

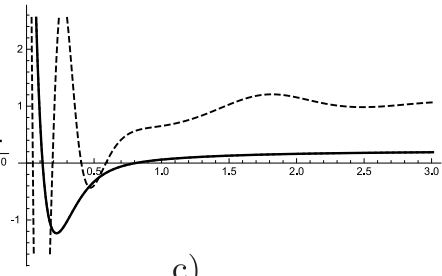

c)

Figure 2. Time-series of the fractional-order Chen system (1)-(3), for $v=0.69$. The step size is $h=0.01$ and $t \in[0,3]$ (FMDTM - dashed line, FAB - solid line, FABM -dotted line]. The integration curves for FAB and FABM are seen as indistinguishable in the given axes scale of the figures. a) time series $x(t) ; \mathrm{b})$ time series $y(t) ; \mathrm{c})$ time series $z(t)$

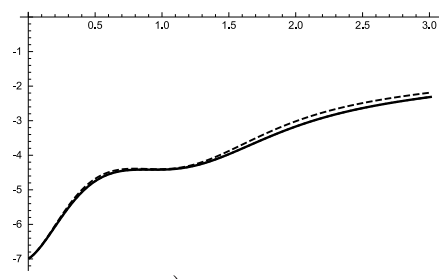

a)

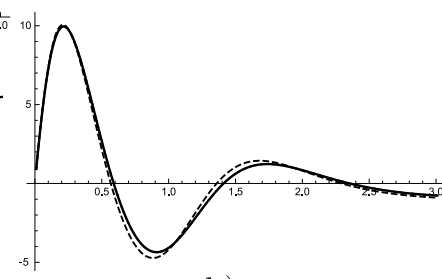

b)

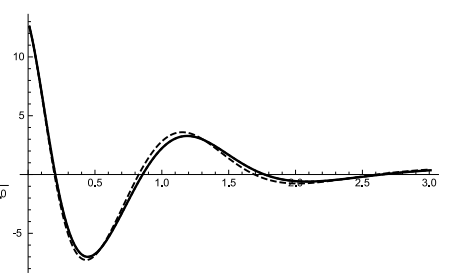

c)

Figure 3. Time-series of the fractional-order Chen system (1)-(3), for $v=0.99$. The step size is $h=0.01$ and $t \in[0,3]$ (FMDTM - dashed line, FAB - solid line, FABM -dotted line]. The integration curves for FAB and FABM are seen as indistinguishable in the given axes scale of the figures. a) time series $x(t)$; a) time series $y(t)$; a) time series $z(t)$

To quantify the deviations of the integration curves obtained by different numerical methods, in Figs. $4-12$ we plot the absolute difference of the different time series. It can be noticed that regardless the value of the fractional-order, the difference of the integration curves obtained by FAB and FABM methods is very small, for example, when $v=0.69$, we have $\operatorname{Abs}[(\mathrm{FABM})-(\mathrm{FAB})] \leq 1.4 \times 10^{-12}$. On the other hand, the integration curves obtained by FMDTM method for $v=0.39$ and $v=0.69$ differ significantly from the FAB and FABM curves [e.g for $v=0.39$, the absolute difference of the $y(t)$ time series between FMDTM and FABM are around 4 (Fig. 5c)]. Nevertheless, the value of the absolute difference becomes significantly lower when $v=0.99$ (Figs. 10-12). 


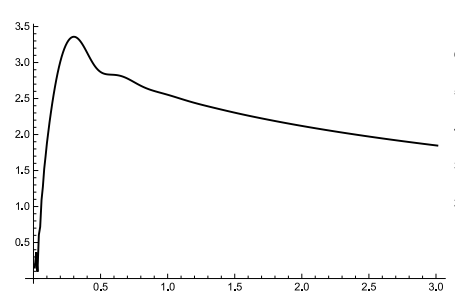

a)

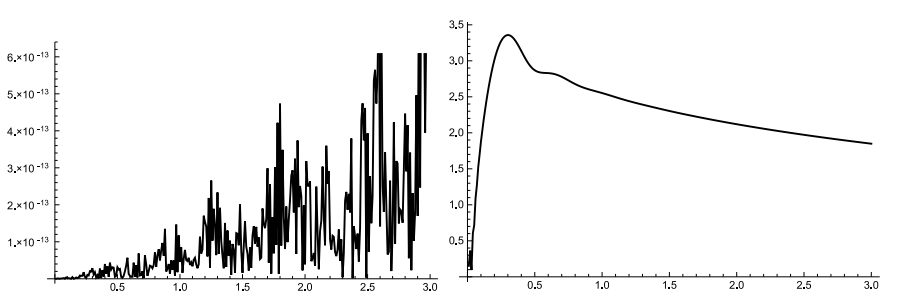

b)

c)

Figure 4. Absolute difference for the time series $x(t)$ of the fractional-order Chen system (1)-(3), for $v=0.39$. The step size is $h=0.01$ and $t \in[0,3]$. a) $\operatorname{Abs}_{x}[(\mathrm{FMDTM})-(\mathrm{FAB})]$; a $)$ $\left.\operatorname{Abs}_{x}[(\mathrm{FABM})-(\mathrm{FAB})] ; \mathrm{a}\right) \operatorname{Abs}_{x}[(\mathrm{FMDTM})-(\mathrm{FABM})]$

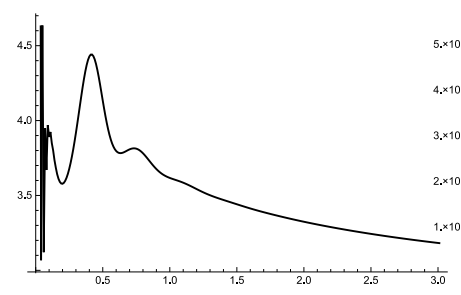

a)

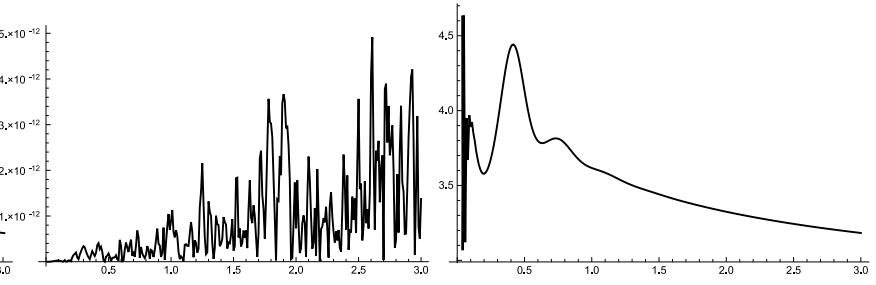

b)

c)

Figure 5. Absolute difference for the time series $y(t)$ of the fractional-order Chen system (1)-(3), for $v=0.39$. The step size is $h=0.01$ and $t \in[0,3]$. a) $\operatorname{Abs}_{y}[($ FMDTM $)-($ FAB $)]$;

b) $\operatorname{Abs}_{y}[(\mathrm{FABM})-(\mathrm{FAB})]$; c) $\operatorname{Abs}_{y}[(\mathrm{FMDTM})-(\mathrm{FABM})]$

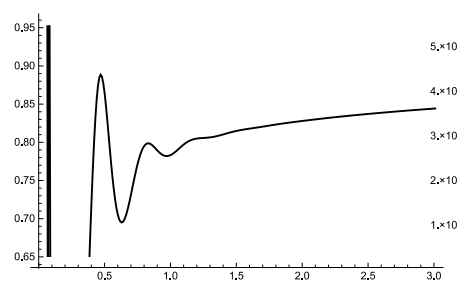

a)

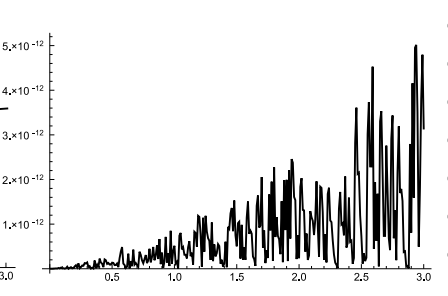

b)

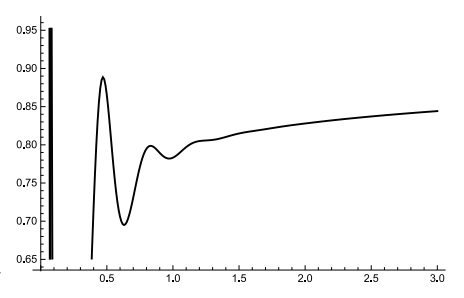

c)

Figure 6. Absolute difference for the time series $z(t)$ of the fractional-order Chen system (1)-(3), for $v=0.39$. The step size is $h=0.01$ and $t \in[0,3]$. a) $\operatorname{Abs}_{z}[($ FMDTM $)-(\mathrm{FAB})]$; b) $\left.\operatorname{Abs}_{z}[(\mathrm{FABM})-(\mathrm{FAB})] ; \mathrm{c}\right) \operatorname{Abs}_{z}[(\mathrm{FMDTM})-(\mathrm{FABM})]$ 
COMPARISON OF DIFFERENT NUMERICAL METHODS FOR FRACTIONAL ... 71

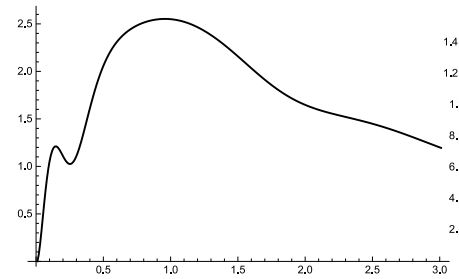

a)

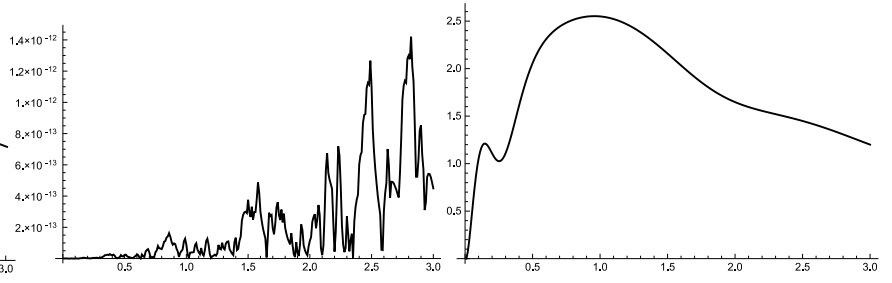

b)

c)

Figure 7. Absolute difference for the time series $x(t)$ of the fractional-order Chen system (1)-(3), for $v=0.69$. The step size is $h=0.01$ and $t \in[0,3]$. a) $\operatorname{Abs}_{x}[(\mathrm{FMDTM})-(\mathrm{FAB})]$; b $)$ $\left.\operatorname{Abs}_{x}[(\mathrm{FABM})-(\mathrm{FAB})] ; \mathrm{c}\right) \operatorname{Abs}_{x}[(\mathrm{FMDTM})-(\mathrm{FABM})]$

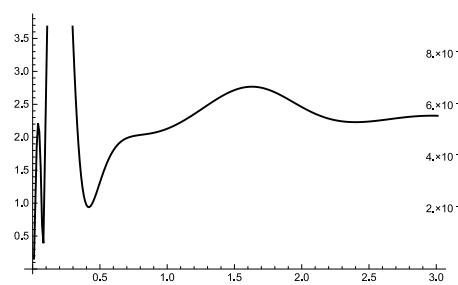

a)

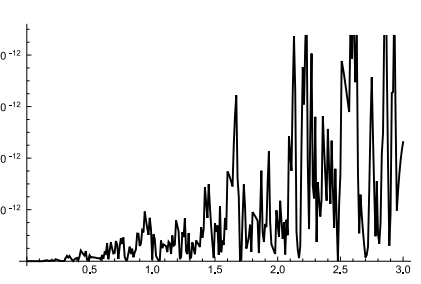

b)

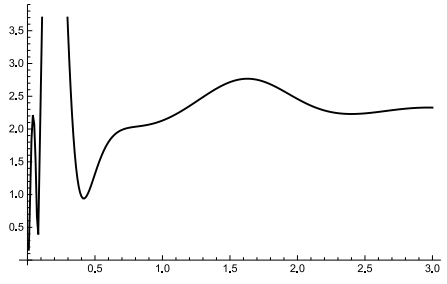

c)

Figure 8. Absolute difference for the time series $y(t)$ of the fractional-order Chen system (1)-(3), for $v=0.69$. The step size is $h=0.01$ and $t \in[0,3]$. a) $\operatorname{Abs}_{y}[($ FMDTM $)-(F A B)]$; b) $\left.\mathrm{Abs}_{y}[(\mathrm{FABM})-(\mathrm{FAB})] ; \mathrm{c}\right) \mathrm{Abs}_{y}[(\mathrm{FMDTM})-(\mathrm{FABM})]$

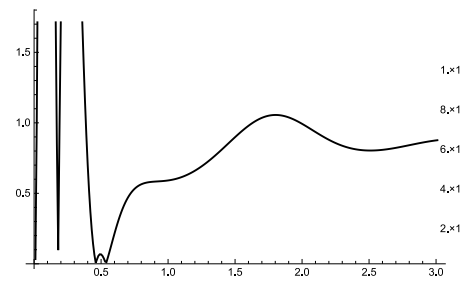

a)

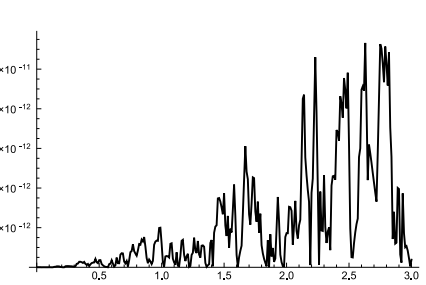

b)

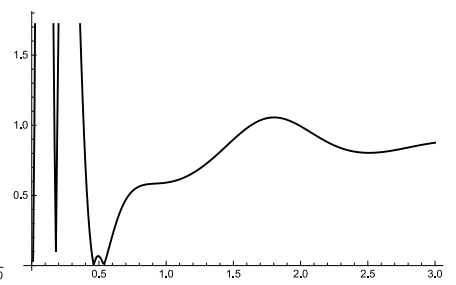

c)

Figure 9. Absolute difference for the time series $z(t)$ of the fractional-order Chen system (1)-(3), for $v=0.69$. The step size is $h=0.01$ and $t \in[0,3]$. a) $\operatorname{Abs}_{z}[(\mathrm{FMDTM})-(\mathrm{FAB})]$; b ) $\left.\mathrm{Abs}_{z}[(\mathrm{FABM})-(\mathrm{FAB})] ; \mathrm{c}\right) \mathrm{Abs}_{z}[(\mathrm{FMDTM})-(\mathrm{FABM})]$ 


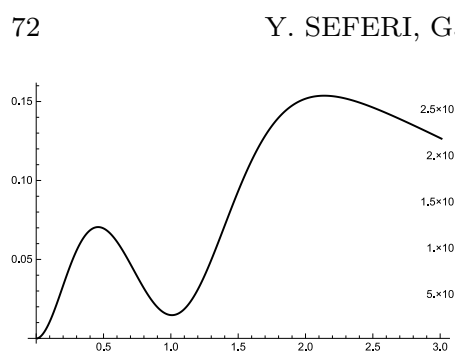

a)

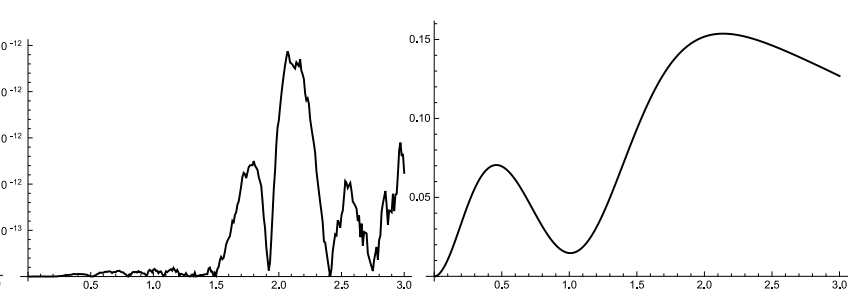

b)

c)

FiguRE 10. Absolute difference for the time series $x(t)$ of the fractional-order Chen system (1)-(3), for $v=0.99$. The step size is $h=0.01$ and $t \in[0,3]$. a) $\operatorname{Abs}_{x}[(\mathrm{FMDTM})-(\mathrm{FAB})]$; b $)$ $\left.\operatorname{Abs}_{x}[(\mathrm{FABM})-(\mathrm{FAB})] ; \mathrm{c}\right) \operatorname{Abs}_{x}[(\mathrm{FMDTM})-(\mathrm{FABM})]$

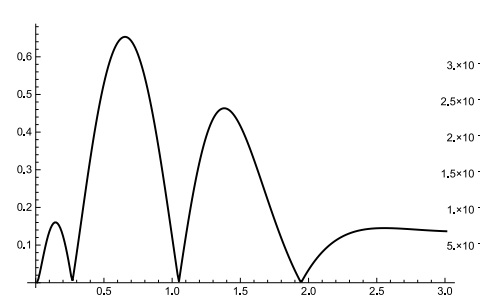

a)

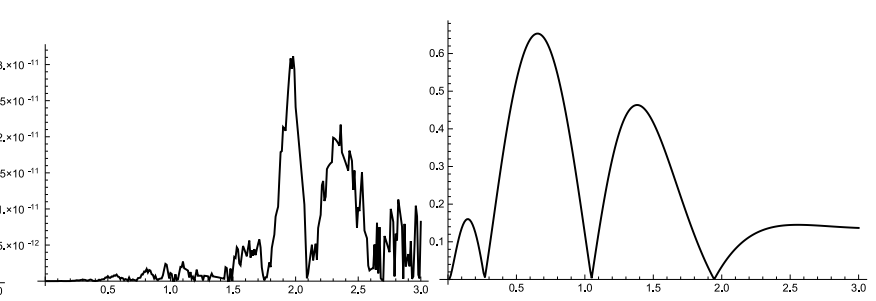

b)

c)

Figure 11. Absolute difference for the time series $y(t)$ of the fractional-order Chen system (1)-(3), for $v=0.99$. The step size is $h=0.01$ and $t \in[0,3]$. a) $\operatorname{Abs}_{y}[(\mathrm{FMDTM})-(\mathrm{FAB})]$; b $)$ $\left.\mathrm{Abs}_{y}[(\mathrm{FABM})-(\mathrm{FAB})] ; \mathrm{c}\right) \mathrm{Abs}_{y}[(\mathrm{FMDTM})-(\mathrm{FABM})]$

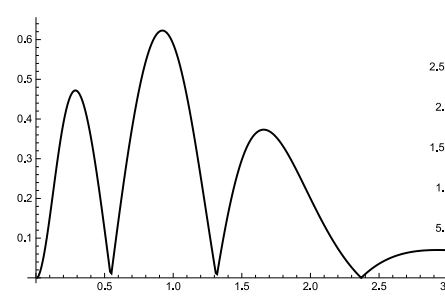

a)

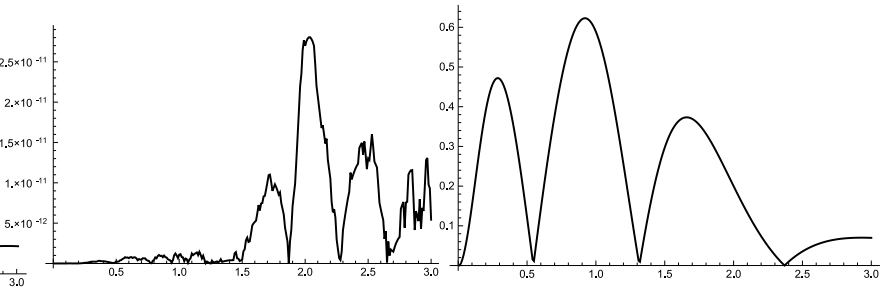

b) c)

Figure 12. Absolute difference for the time series $z(t)$ of the fractional-order Chen system (1)-(3), for $v=0.99$. The step size is $h=0.01$ and $t \in[0,3]$. a) $\operatorname{Abs}_{z}[(\operatorname{FMDTM})-(\mathrm{FAB})]$; b ) $\left.\operatorname{Abs}_{z}[(\mathrm{FABM})-(\mathrm{FAB})] ; \mathrm{c}\right) \mathrm{Abs}_{z}[(\mathrm{FMDTM})-(\mathrm{FABM})]$ 


\section{Conclusions}

In this paper three different numerical approximation schemes (FAB, FABM and FMDTM) have been applied to find the time-series solutions of the fractionalorder Chen system. We have aimed to quantify the distinction between the integration methods by depicting the time series of the absolute difference for the same system parameters and initial conditions, by varying the order of the fractional derivative $v$.

The results show that FAB and FABM methods are in excellent agreement at each value of the fractional-order $v$, but they both generally differ from the time series approximations obtained by FMDTM. We have shown numerically that this difference is less pronounced as the value of the fractional-order becomes closer to one.

Clearely, the methods of numerical integration are substantially different with respect to the speed of the numerical computation, since FMDTM is using the time-series values of the previous step as an input for computing the

next values, whilst FAB requires a knowledge of the whole history to compute the next step, and thus is much time-consuming with respect to FMDTM.

\section{REFERENCES}

[1] C. Bervillier, Status of the diffrential transformation method, Applied Mathematics and Computation, 20, (2012), 10158-10170.

[2] A. K. Alomari, A new analytic solution for fractional chaotic dynamical systems using the differential transform method, Computers and Mathematics with Applications, 61, (2011), 2528-2534.

[3] K. Diethelm, N. J. Ford and A. D. Freed, Detailed error analysis for a fractional Adams method, Numerical Algorithms, 36, (2004), 31-52.

[4] S. V. Ertürk, Sh. Momani, Solving systems of fractional differential equations using differential transform method, Journal of Computational and Applied Mathematics, 215, (2008), 142-151.

[5] D. Baleanu, K. Deithelm, E. Scalas, J. J. Trujillo, Fractional Calculus: models and numerical methods, (2017), World Scientific, Singapore.

[6] R. Garrappa, On some explicit Adams multistep methods for fractional differential equations, Journal of Computational and Applied Mathematics, 229, (2009), 392-399.

[7] L. Changpin, Z. Fanhai, Numerical Methods for Fracional Calculus, (2015), CRC Press Taylor and Francis Group, New York.

[8] G. Markoski, Bifurcation Analysis of fractional-order Chaotic Rossler System, Matematichki Bilten, 42, (2018), 28-37.

[9] K. Diethelm, N. J. Ford and A. D. Freed, A Predictor-Corrector Approach for the Numerical Solution of Fractional Differential Equations, Nonlinear Dynamics, 29, (2001), $3-22$. 
[10] S. K. Miller, B. Ross, An introduction to the fractional calculus and fractional differential equations, (1993), John Wylei and Sons, Inc., Canada.

[11] Lu J., Chen G., Cheng D., Celikovski S., Bridge the gab between Lorenz system and the Chen system, International Journal of Bifurcation and Chaos, 12, (2002), 2917-2926.

[12] Alomari A. K., A novel solution for fractional chaotic Chen system, J. Nonlinear Sci. Appl., 8, (2015), 478-488.

[13] S. A. Efteklari, A. A. Jafari, Numerical simulation of chaotic dynamical systems by the method of differential quadrature, Scientia Iranica B, 19(5), (2012), 1299-1315.

University of Tetova, Macedonia

Email address: ylldrita.seferi@unite.edu.mk

Institute OF Mathematics,

Faculty of Natural Sciences and Mathematics,

Ss. Cyril and Methodius University, Skopje, Macedonia

Email address: gorgim@pmf .ukim.mk

INSTITUTE OF PHISICS,

Faculty of Natural Sciences and Mathematics,

Ss. Cyril And Methodius University, Skopje, Macedonia

Email address: agjurcin@yahoo.com 